\title{
Chromatin loop anchors are associated with genome instability in cancer and recombination hotspots in the germline
}

Vera B. Kaiser ${ }^{*}$ (D) and Colin A. Semple

\begin{abstract}
Background: Chromatin loops form a basic unit of interphase nuclear organization, with chromatin loop anchor points providing contacts between regulatory regions and promoters. However, the mutational landscape at these anchor points remains under-studied. Here, we describe the unusual patterns of somatic mutations and germline variation associated with loop anchor points and explore the underlying features influencing these patterns.

Results: Analyses of whole genome sequencing datasets reveal that anchor points are strongly depleted for single nucleotide variants (SNVS) in tumours. Despite low SNV rates in their genomic neighbourhood, anchor points emerge as sites of evolutionary innovation, showing enrichment for structural variant (SV) breakpoints and a peak of SNVs at focal CTCF sites within the anchor points. Both CTCF-bound and non-CTCF anchor points harbour an excess of SV breakpoints in multiple tumour types and are prone to double-strand breaks in cell lines. Common fragile sites, which are hotspots for genome instability, also show elevated numbers of intersecting loop anchor points. Recurrently disrupted anchor points are enriched for genes with functions in cell cycle transitions and regions associated with predisposition to cancer. We also discover a novel class of CTCF-bound anchor points which overlap meiotic recombination hotspots and are enriched for the core PRDM9 binding motif, suggesting that the anchor points have been foci for diversity generated during recent human evolution.
\end{abstract}

Conclusions: We suggest that the unusual chromatin environment at loop anchor points underlies the elevated rates of variation observed, marking them as sites of regulatory importance but also genomic fragility.

Keywords: Cancer, Recombination, DNA breakage, Hi-C, Chromatin loops

\section{Background}

Recent evidence shows that many cancers and developmental disorders involve disruptions of chromatin organisation. Insertions and deletions are reported to alter the boundaries of topologically associating domains (TADs), which normally constrain the regulatory interactions of resident promoters and enhancers, causing dysregulated gene expression [1, 2]. Disruptions of particular TAD boundaries have been reported in neuroblastoma $[3,4]$, medulloblastoma [5], leukaemia [6, 7] and other cancers [8], consistent with the hypothesis that structural variants (SVs) remodelling TAD boundaries may act as

\footnotetext{
* Correspondence: vera.kaiser@igmm.ed.ac.uk

MRC Human Genetics Unit, MRC Institute of Genetics and Molecular Medicine, University of Edinburgh, Western General Hospital, Crewe Road, Edinburgh EH4 2XU, UK
}

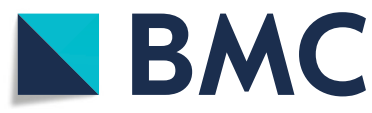

(C) The Author(s). 2018 Open Access This article is distributed under the terms of the Creative Commons Attribution 4.0 International License (http://creativecommons.org/licenses/by/4.0/), which permits unrestricted use, distribution, and

reproduction in any medium, provided you give appropriate credit to the original author(s) and the source, provide a link to the Creative Commons license, and indicate if changes were made. The Creative Commons Public Domain Dedication waiver (http://creativecommons.org/publicdomain/zero/1.0/) applies to the data made available in this article, unless otherwise stated.

oncogenic 'driver' mutations under selection in tumour cells [9].

CTCF plays important roles in chromatin organisation, both demarcating domain boundaries as an insulator element $[10,11]$ and by bringing DNA sites that are distant in linear genomic distance intro close spatial proximity [12]. According to the loop extrusion model, it is proposed that pairs of CTCF binding sites may physically interact to form anchoring sites at the base of a chromatin loop, acting as physical barriers to the ring-shaped cohesin complex, which slides along the DNA [13-15]. Topological stress relief at loop anchor points may be provided by TOP2B [16, 17], an enzyme that transiently creates double-strand breaks (DSBs) and re-joins the DNA in a different spatial configuration, and TOP2B binding sites in the breast cancer cell line MCF-7 have been shown to be co-located with CTCF 
motifs [18]. On a larger scale, complex arrays of DNA loops are thought to make up the substructure of regulatory domains such as TADs [19], and recent experiments highlight the critical importance of CTCF for loop and TAD formation [20].

CTCF binding sites are highly mutated across cancer types, especially when they are located within loop anchor points (LAPs) [21, 22]. Hyper-methylation of the GC-rich CTCF binding motif has been shown to reduce CTCF binding in glioma, leading to the up-regulation of known oncogenes [23]. Hnisz et al. [7] have shown that constitutive CTCF-CTCF binding site interactions delineating loops are recurrently deleted in T-cell acute lymphoblastic leukaemia, which leads to oncogene activation. Overall, these data suggest that domain boundary or LAP lesions affecting gene regulation are far from rare in cancers and occur at comparable rates to recurrent in-frame gene fusions [8]. However, it is unclear whether LAPs are intrinsically prone to high mutation rates in cancer, constituting a novel class of fragile sites in the genome, or whether the observed lesions affecting LAPs confer a selective advantage to tumour cells.

Somatic mutation rates vary across the genome, and a large fraction of this variation can be attributed to differences in replication timing, with late replicating regions of the genome accumulating increased levels of single nucleotide variants (SNVs) [24]. Large regions of chromosomes (encompassing hundreds of kilobases) are replicated synchronously in replication domains that correspond closely to TADs, linking chromatin organisation to spatiotemporal variation in replication [25], while other, inter-correlated features of chromatin, such as histone methylation or acetylation patterns, are also associated with somatic mutation rates [26]. On a much finer scale, the individual binding sites of a variety of DNA binding factors, including CTCF, appear to obstruct the lagging strand replication and DNA repair machinery and induce higher mutation rates in human and yeast [27-29]. However, the mutational landscape associated with intermediate levels of chromatin organisation, such as chromatin loops, are not well studied.

Similarly, the influence of genomic features on structural rearrangements in cancer is relatively under-studied, but it appears that different cancer types follow different patterns. For some cancer types, such as breast cancer, structural somatic variants are enriched within early replicating, GC-rich, transcribed regions of the genome, whereas the opposite trend was observed for cancers such as prostate and melanoma [30]. Further, the 3D structure of the genome may predispose regions of the genome that are in $\mathrm{Hi}-\mathrm{C}$ contact to be more likely to undergo structural rearrangements $[31,32]$.

A cellular process intrinsically linked to double-strand breakage is genetic recombination, which is involved in
DNA repair in the somatic cell and is an essential process in the production of germ cells. Replication- and recombination-associated mechanisms are hypothesised to lead to the formation of structural variants and may, therefore, contribute to structural variation in cancers [33].

During meiosis, recombination is initiated by DSBs and occurs non-randomly across the genome; it is at its highest level at recombination hotspots (HSs) where the majority $(60 \%)$ of recombination events take place [34, 35]. While it is known that recombination produces large SVs, the effect of recombination on the emergence of SNVs is less clear-as is its relation to chromatin structure. There is evidence that recombination is mutagenic in yeast [36, 37], and a recent study of 283 human trios has shown a correlation between the rate of recombination events in parental germ cell genomes and the rate of de novo SNVs in offspring genomes, suggesting a mutagenic effect of recombination [38]. However, the data supporting this were necessarily sparse, given the low de novo mutation rates in the germline.

Here, we explore the genomic landscape around LAPs and demonstrate that the unusual chromatin environment at LAPs is matched by unexpected mutation rates, establishing LAPs as foci of evolutionary change and fragile sites in cancer.

\section{Results}

Previous work has demonstrated elevated SNV rates at CTCF binding sites within LAPs in a variety of cancers [21]. This motivated us to investigate genome-wide somatic mutation rates around CTCF-containing LAPs from the aggregated Hi-C datasets of Rao et al. [19] (see "Methods"), using 13 recently released ICGC somatic variant datasets ascertaining both SNVs and SVs in nine different tumour types [39]. Within $50 \mathrm{~kb}$ of LAPs, the ICGC pan-cancer samples show a dramatic drop in SNV rates (Fig. 1a). This regional decrease in SNVs at LAPs is in stark contrast to the high mutation rate observed at the short 19-bp CTCF-binding motifs located inside LAPs, which is 12.3 $\mathrm{SNVs} / \mathrm{Mb}^{-1}$, or more than three times higher than the local background mutation rate. Plotting SNV rates at 20-bp resolution, a peak of SNVs in the centre of the LAPs at CTCF-binding sites becomes apparent (as seen in Kaiser et al. [21]; Fig. 1b and Additional file 1: Figure S1), as well as a periodic pattern of mutation reflecting nucleosome occupancy $[28,40]$. Thus, CTCF-binding sites within LAPs are prone to local somatic hypermutation in tumours but often reside within broader genomic regions with significantly reduced SNV rates.

\section{Chromatin loop anchors are HSs of structural variation in tumours}

In contrast to SNVs, the frequency of pan-cancer SV breakpoints shows a significant increase at LAPs, inverting the 

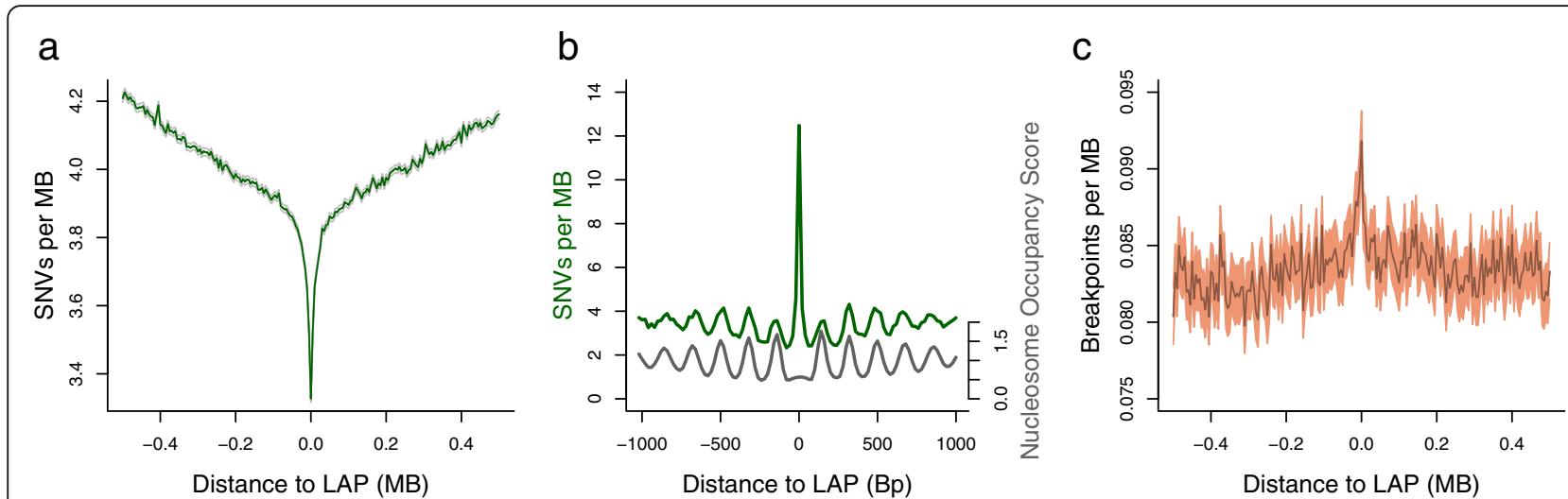

Fig. 1 Loop anchor points are sites of genomic instability in cancer. a Average SNV rates per tumour within 500 kb of all LAPs. b Finer scale average SNV rates per tumour (green line) within $1 \mathrm{~kb}$ of LAPs, centred on the CTCF site within each LAP, with nucleosome occupancy based on MNase-seq data over the same intervals (grey line). c Average SV breakpoint rates per tumour within 500 kb of all LAPs. The 95\% confidence intervals, calculated assuming a Poisson distribution of mutation rates, are shown as shaded regions in a and $\mathbf{c}$. Note that the $y$-axis scaling has been selected to clearly depict statistically significant trends in these data

pattern seen for SNVs over the same range of flanking sequence (Fig. 1c). This shows that LAPs are structurally fragile sites in cancer, and so we examined associations between LAPs and more direct measures of genomic instability. Lensing et al. [41] identified genome-wide foci of endogenous DSBs in vitro and these sites show a striking 3.7-fold enrichment at LAPs compared to their flanking regions (Fig. 2a). A proportion of this enrichment may be attributable to the close proximity of LAPs to promoters and enhancers, which are known to suffer elevated DSB rates [41]. However, LAPs lacking any overlap with known promoters and enhancers show similarly elevated rates to those that do (Fig. 3). Consistent with inherent genomic instability, LAPs are also enriched in predicted G-quadruplexes (G4s), a DNA secondary structure associated with regulatory regions and DSB formation in cancers [42] (Fig. 2b).

BRCA1 and BRCA2 are two well-characterised tumour suppressor genes involved in DSB repair by homologous recombination [43-45]. BRCA1 is often recruited to sites of active transcription, which are prone to DNA damage during the formation of transcriptional R-loops [46]. We found a strong enrichment of BRCA1 at HMEC LAPs in MCF10A, a normal breast epithelial cell line, as well as increased RAD51 binding-which mediates BRCA2 binding-around LAPs in the MCF-7 cell line (Fig. 2c); this is, to our knowledge, the first observation of BRCA1/2 association with LAPs, albeit this appears to be driven by neighbouring active promoters and enhancers rather than the LAPs themselves (Additional file 1: Table S1).

\section{LAPs overlap recombination HSs in human populations}

Intriguingly, LAPs show an unexpected genome-wide correspondence with germline recombination HSs, calculated from genotyping of extant human populations [47], such that $16 \%$ of LAPs overlap HSs (based upon 100,000 circular permutations; $p<10^{-5}$; Fig. $\left.4 \mathrm{a}\right)$. These overlaps are notably precise, so that the association between LAPs and HSs drops when the two sets of regions are shifted with respect to each other by less than $50 \mathrm{~kb}$ (Fig. 4b). Thus, their correspondence is not simply attributable to the enrichment of both sets of features within certain broader neighbourhoods, such as replication timing domains or nuclear compartments. Recombination HSs are known to often contain the motif bound by PRDM9, a critical component of the recombination machinery $[48,49]$, and, using stringent search criteria (see "Methods"), we find this motif in 17\% of HSs. Similarly, we find that $13 \%$ of LAPs also contain at least one PRDM9 core motif, which is an enrichment of $~ 33 \%$ compared to the median number of motifs per $5-\mathrm{kb}$ bin in LAP flanking regions (Fig. 4c) and also constitutes a significant enrichment genome-wide (circular permutations in $R$ : $\left.p<10^{-5}\right)$. For the $16 \%$ of LAPs directly overlapping HSs (HS-LAPs) we find no further enrichment of the PRDM9 motif (13.6\% of HS-LAPs contain the motif), but, as expected, there is a notable increase in the recombination rate at HS-LAPs (Fig. 4d). This is consistent with dual roles for a subset of LAPs, both as units of chromatin organisation and as HSs of structural variation.

The recombination enzyme PRDM9 is expressed exclusively in testis, but it is also expressed in a variety of cancer cell lines and samples and has been proposed as a cancer biomarker [50]. We observe modestly increased SNV rates at recombination HSs in cancer (Fig. 5a) but do not find any pan-cancer increase in SV breakpoints around HSs (Additional file 1: Figure S2), which might be expected if meiotic recombination complexes were activated in the tumours examined here. In addition, the 
a

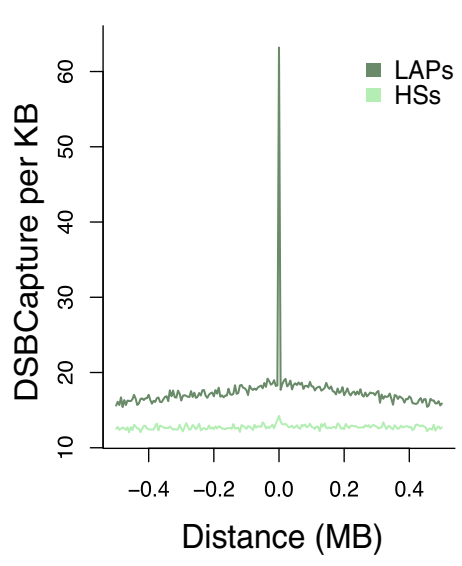

$\mathrm{C}$

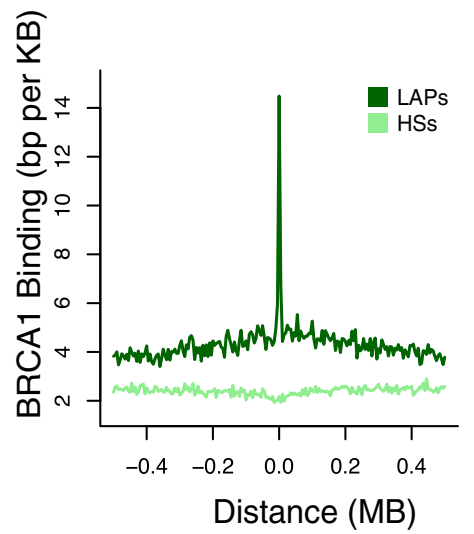

$\mathrm{b}$
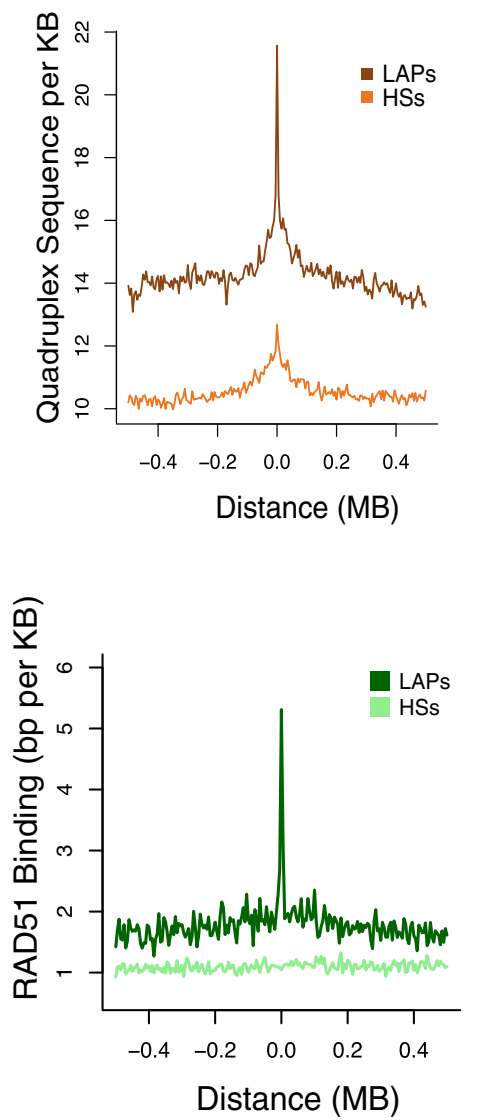

Fig. 2 Loop anchor points are susceptible to somatic double-strand breaks. a DNA double-strand break density in the NHEK cell line, within $500 \mathrm{~kb}$ of NHEK LAPs and recombination HSs. b Predicted quadruplex structure forming sequence density within $500 \mathrm{~kb}$ of the union set of LAPs. c Average density of BRCA1 ChIP-seq binding peaks for MCF-10A cells and RAD51 ChIP-seq binding peaks for MCF-7 near HMEC LAPs and recombination HSs
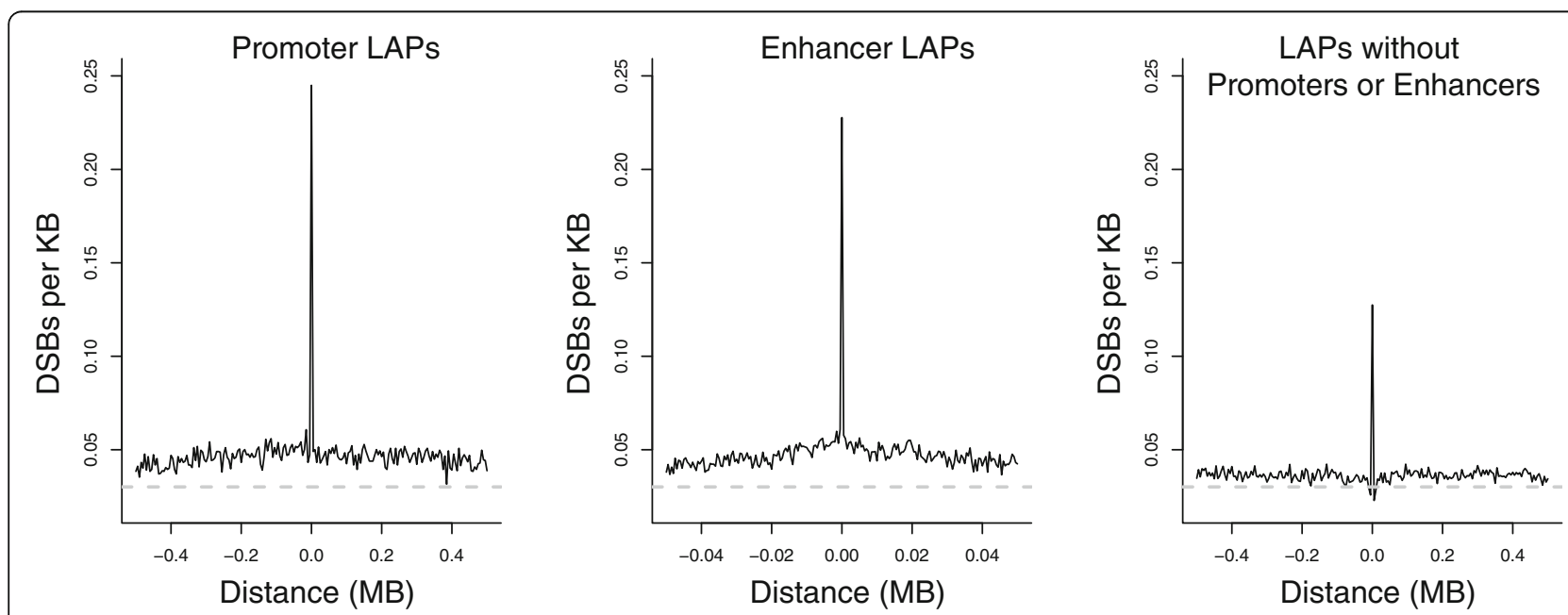

Fig. 3 Rates of spontaneous DSBs increase at LAPS, also when no promoter or enhancer sequence is present. The overlap, per kilobase, with NHEK DSB regions is shown for NHEK LAPs that overlap the following ENCODE chromHMM annotations: NHEK promoters (762 LAPs), NHEK enhancers (1485 LAPs), or neither NHEK promoters or NHEK enhancers (2030 LAPs). The dashed line indicates the genome-wide average 
a

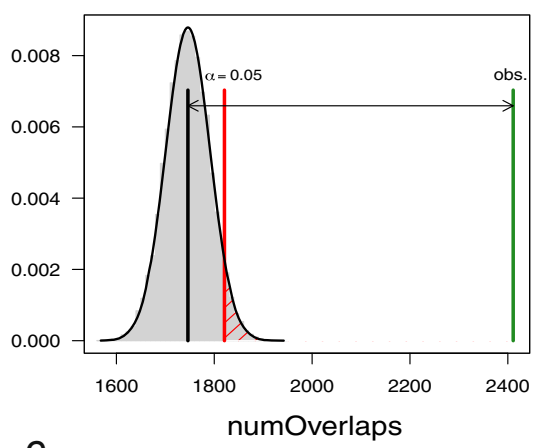

C

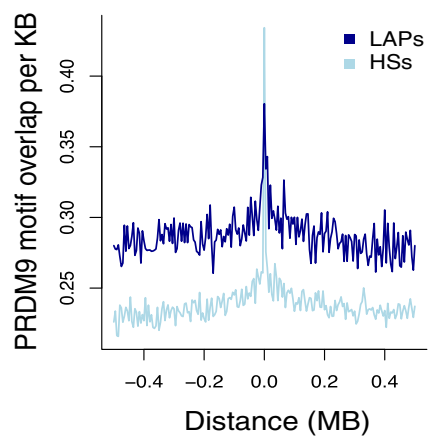

b

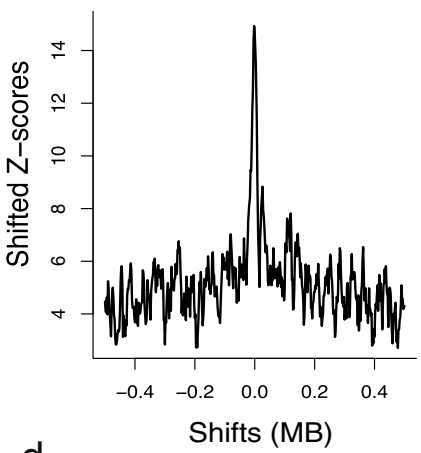

d

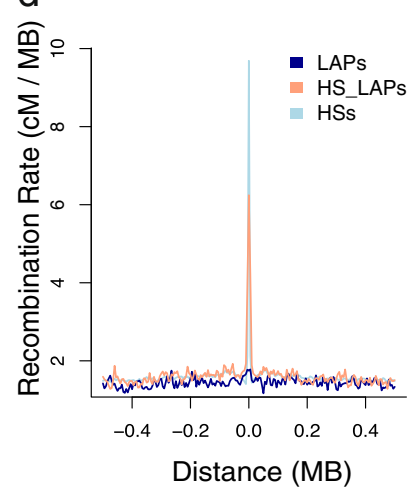

Fig. 4 LAPs and recombination HSs are physically linked. a Probability density plot showing the observed number (green line) versus expected distribution (grey histogram) of overlaps between LAPs and HSs, $p<10^{-5}$ based upon 100,000 circular permutations. The threshold for statistical significance is indicated by the red line. $\mathbf{b}$ Variation in circular permutation z-scores ( $y$-axis) relative to shifts in the location of HSs ( $x$-axis),

suggesting that significance of overlaps between LAPs and HSs results from specific local (not broader regional) overlaps. c PRDM9 binding motif density, which is targeted by the recombination machinery, within $500 \mathrm{~kb}$ of recombination HSs and LAPs. $\mathbf{d}$ Average recombination rates within $500 \mathrm{~kb}$ of recombination HSs, the subset of LAPs overlapping HSs (HS_LAPs) and all LAPs

histone modification H3K4me3, which is deposited by PRDM9 at DSBs, is not observed at recombination HSs in the cancer cell lines HepG2 and MCF-7 (Additional file 1: Figure S3). In contrast, H3K4me3 increases around LAPs (Additional file 1: Figure S3), possibly as a result of PRDM9 recruitment to the PRDM9 motifs enriched at LAPs or, more likely, because H3K4me3 is a mark of active promoters enriched at chromatin boundaries [11]. We cannot, however, exclude the possibility that PRDM9 is active in at least a subset of the tumours
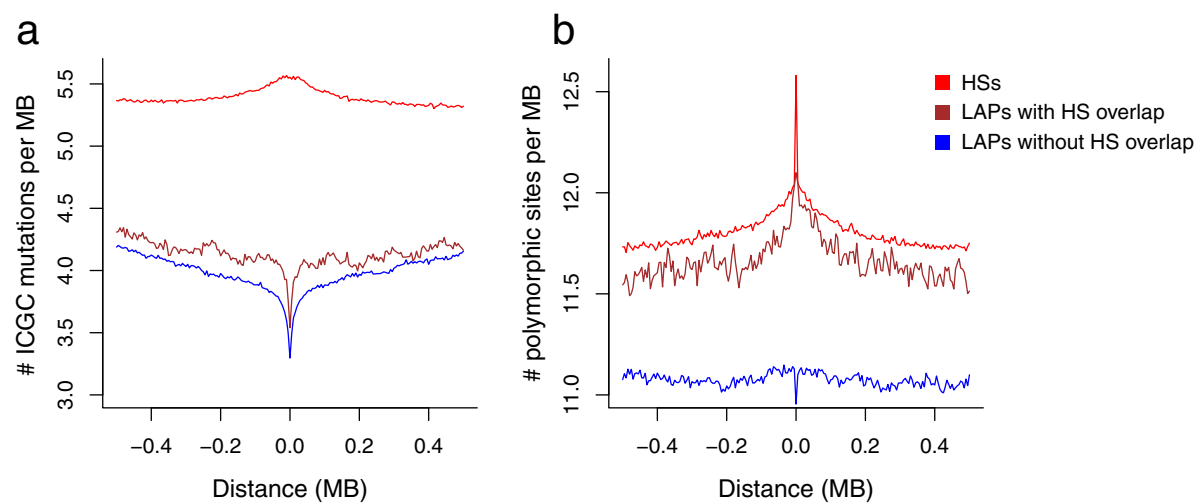

Fig. 5 The impact of loop anchor points on tumour and population variation. a Average SNV somatic mutations based on ICGC pan-cancer tumour sequencing data and $\mathbf{b}$ the average number of polymorphic segregating sites within $500 \mathrm{~kb}$ of LAPs and HSs. LAPs are subdivided into those that do or do not overlap recombination HSs, respectively 
under investigation and contributes to the increase in SV rates at LAPs.

A substantial fraction of LAPs ( $47 \%$ of those studied here) constitute regulatory domain boundaries [19], while an even higher proportion, 69\%, overlap DSB-foci [41], and 16\% overlap recombination HSs (Table 1). Genome-wide, however, these three categories of LAPs appear to be largely independent, as the extent of overlap between categories was remarkably similar to the expected rate assuming independent distributions across the genome. For example, LAPs that appear as domain boundaries were as likely to overlap recombination HSs as LAPs that do not act as boundaries (Table 1). There was also no enrichment of Gene Ontology (GO) terms associated with genes neighbouring HS-LAPs versus a background set of genes found at all LAPs, i.e. HS-LAPs are not found near specific functional categories of genes.

LAPs are associated with similar mutational landscapes in tumours, irrespective of whether they overlap HSs or locate outside high recombination regions: both types of LAP show a distinctive dip in tumour SNV rates (Fig. 5a), while cancer mutation rates somewhat increase around recombination HSs, by $~ 3.5 \%$ compared to the median rate within the flanking regions. As expected, recombination HSs are associated with a pronounced increase in SNPs in the 1000 Genomes Project (1KG) dataset [47], and LAPs that overlap HSs are also enriched for segregating variants, by $\sim 7 \%$ compared to the flanking regions (Fig. 5b). Accordingly, population genetic processes that increase variation at HSs-such as selective sweeps and reductions in background selection [51]-also appear to have impacted germline variation at HS-LAPs. However, germline de novo SNV rates at LAPs are not reduced as they are in cancer and, similarly, we do not observe an increase in SVs near HSs in cancer (Additional file 1: Figure S2), suggesting fundamentally different influences on germline mutation rates versus cancer-associated somatic mutation rates at these sites.

\section{Cancer mutation rates at CTCF sites outside LAPs and at non-CTCF LAPs}

To investigate if the observed mutational patterns at LAPs are due to the presence of CTCF binding alone, we also studied mutational patterns around constitutive CTCF-binding sites located outside the known set of LAPs, i.e. sites that may not act as anchor points. Compared to the CTCF-LAPs, we observe a similar decrease of SNV rates in the 1-Mb sequence surrounding CTCF sites (Fig. 6) and a less distinct peak of mutation when zooming in to high resolution (Additional file 1: Figure S4). However, we note that an unknown proportion of these CTCF sites may also be involved in loop formation but were not detected in present LAP data-either because they participate in transient or cell type-specific LAPs or because the sequencing depth was insufficient in the original cell lines. Indeed, the available data suggest that LAP detection is a linear function of $\mathrm{Hi}-\mathrm{C}$ sequencing depth and many loops remain to be discovered (Additional file 1: Figure S5). Non-LAP CTCF sites also showed an increased rate of DNA breakage (Fig. 6b) and a strong enrichment of BRCA1 (Additional file 1: Figure S4), consistent with general co-binding of these factors and recent data which suggest that CTCF has roles in regulating the homologous recombination repair

Table 1 Overlap of LAPs with domain boundaries, recombination HSs and DSB-prone regions

\begin{tabular}{|c|c|c|c|c|}
\hline a) Feature & Feature count & Genomic span (Mb) & & \\
\hline LAPS & 14,737 & 73.69 & & \\
\hline Boundaries & 40,615 & 250.89 & & \\
\hline HSs & 32,984 & 181.79 & & \\
\hline DSB-prone regions & 84,946 & 34.69 & & \\
\hline b) Intersection & Number of LAPs & Fraction of LAPs & Expected fraction (circular permutations) & $p$ value \\
\hline LAPs $\cap$ Boundaries & 6960 & 0.47 & 0.15 & $<10^{-4}$ \\
\hline LAPs $\cap \mathrm{HSS}$ & 2385 & 0.16 & 0.12 & $<10^{-4}$ \\
\hline LAPs $\cap$ DSB-prone regions & 10,133 & 0.69 & 0.14 & $<10^{-4}$ \\
\hline c) Intersection & Number of LAPs & Fraction of LAPs & Expected fraction (feature intersection) & $p$ value \\
\hline LAPs $\cap \mathrm{HSs} \cap$ DSB-prone regions & 1709 & 0.12 & 0.11 & $<0.001$ \\
\hline LAPs $\cap$ HSs $\cap$ boundaries & 1172 & 0.08 & 0.08 & $<0.05$ \\
\hline LAPs $\cap$ HSs $\cap$ boundaries $\cap$ DSB-prone regions & 836 & 0.06 & 0.05 & $<0.05$ \\
\hline
\end{tabular}

a The number of features and their genomic span. $\mathbf{b}$ The number of LAPs that overlap with domain boundaries, HSs or DSB-prone regions, as well as the fraction of unique LAPs in each intersection (as a proportion of the total dataset of 14,737 LAPs). The expected fraction (column 4) and the corresponding $p$ value (column 5) were calculated based on 10,000 circular permutations in R. c Columns 2 and 3 as in b. The expected fraction (column 4) was calculated by multiplying the respective fractions shown in column 3 of $\mathbf{b}$. For example, the fraction of LAPs in the intersection "LAPs $\cap$ HSs $\cap$ DSB-prone regions" is equal to $0.16 \times 0.69=0.11$. The hypergeometric test was used to assess the statistical significance of overlap (column 5) 

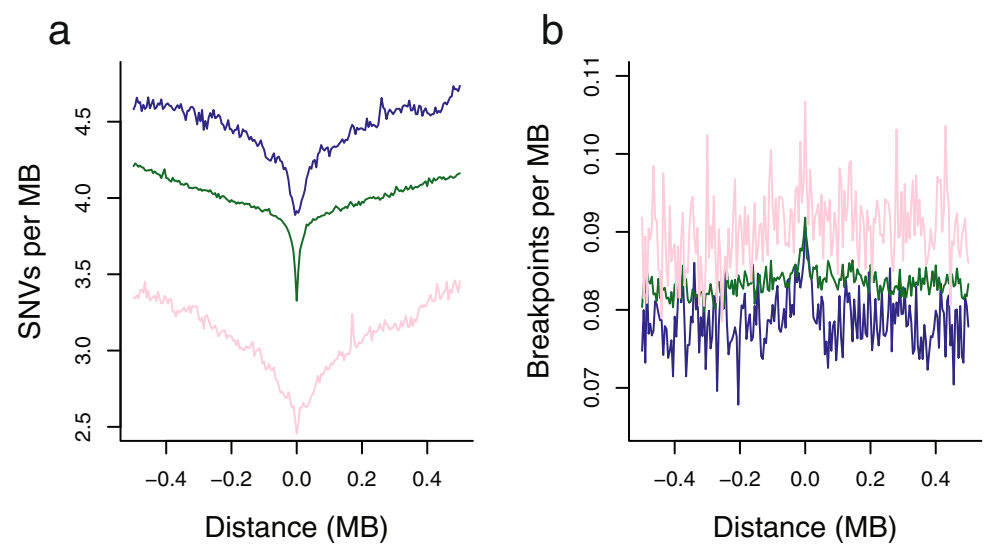

non-CTCF LAPS

LAP CTCFS

CTCF motifs

outside LAPS rates. The central $5-\mathrm{kb}$ windows of all three plots in $\mathbf{b}$ are statistically elevated compared to the flanking regions (resampling one million times with replacement; $p<0.01$ )

pathway [52], as well as a high degree of genome fragility for these non-LAP CTCF sites.

Conversely, we considered a stringent set of 2102 LAPs that are devoid of CTCF motifs, with no evidence for CTCF ChIP-seq binding across ENCODE datasets. With respect to mutation rates, these non-CTCF LAPs behave similarly to CTCF-containing LAPs and contain similar mutational signatures to CTCF-LAPs (Fig. 6 and Additional file 1: Figure S6), while CTCF motifs inside CTCF-LAPs showed no evidence for specific mutational signatures associated with processes such as APOBEC editing after single-strand DNA exposure [53, 54] (Additional file 1: Figure S7). This suggests that the genome architecture or other common features of LAPs impact on their propensity to mutation, such as the presence of enhancer-promoter loops [16].

The most common motifs at non-CTCF LAPs were the promoter-associated motifs MA0506.1 (NRF1), MA0516.1 (SP2), and MA0079.3 (SP1), but none of these motifs showed any enrichment compared to CTCF-binding LAPs. Interestingly, the overlap between recombination HSs and LAPs is confined to LAPs that bind CTCF and is not found for the stringently defined set of 2102 non-CTCF LAPs. Only $11 \%$ of non-CTCF LAPs overlap HSs (not significant by circular permutations) and only $5 \%$ contain the PRDM9 motif, which is a significant depletion compared to expectation $(p=0.0044)$.

\section{Chromatin features influence increased mutation rates at LAPs}

LAPs are relatively GC-rich, enriched for histone modifications associated with active transcription (H3K27ac, H3K27me3, H3K36me3, H3K4me3 and DNase sensitive open chromatin) and depleted for the repressive mark H3K9me3; they also tend to be found in relatively early replicating regions of the genome (Additional file 1: Figure S3).
Recombination HSs also show locally increased GC content, but otherwise possess a contrasting set of features, consistent with their presence in later replicating regions (Additional file 1: Figure S3). Accordingly, LAPs tend to be enriched for genes and actively transcribed regions [19], whereas HSs are located, on average, further away from genic sequence. This raises the possibility that the unusual mutational properties of LAPs may be explained by their distinctive chromatin and sequence features.

To investigate this further, we first identified the specific tumour type most suited for an in-depth analysis. Additional file 1: Figure S8 shows SNV and SV rates around LAPs for all nine cancer types in this study separately. SNV rates are consistently reduced near LAPs in several tumour types, but there are exceptions, such as the malignant lymphoma (MALY-DE) dataset, which does not show a pronounced dip in mutation rates near LAPs even though it includes a large number of SNVs (Additional file 1: Table S2). A pan-cancer analysis is best suited to highlight general patterns, but, for SVs in particular, stratifying mutations by tumour type reduces the power to detect any patterns on a per-tumour basis as SVs are, on average, $~ 100$-fold less frequent than SNVs (Additional file 1: Table S2). Beyond differences in dataset sample sizes, the variability among tumour types most likely reflects the limitations of the current $\mathrm{Hi}-\mathrm{C}$ data, which may be poorly matched to the cells in some cancer samples. However, we could make use of the high-resolution mammary epithelium LAPs from the Rao et al. [19] dataset, which are well matched to the breast cancer data, and a range of epigenetic information is available for this tissue type; note that breast cancer mutations showed both a clear dip in SNV rates and a peak of SV rates near LAPs (Additional file 1: Figure S8). 
Accordingly, we assessed the extent to which mammary epithelium-derived chromatin features (from the MCF-7 and MCF10A cell lines) and a variety of other cell type-specific features were associated with mutation rates in a large ICGC breast tumour dataset (BRCA-EU). Specifically, we used random forest regression to construct models of mutation rates observed within all 5-kb windows from the 500-kb regions flanking all mammary epithelium LAPs (derived from HMEC cell line $\mathrm{Hi}-\mathrm{C}$ data) plus the 5-kb LAP regions themselves ("Methods"). Similar models have previously shown high predictive accuracy in modelling aspects of nuclear organisation and provide variable importance estimates that are robust to the inter-correlated nature of chromatin feature input variables [11]. In our model, by far the most important predictor of the BRCA-EU SNV rate was replication timing, with reduced levels of mutation observed in early replicating regions (Fig. 7), consistent with other studies of breast cancer mutation patterns [40]. The correlation coefficient between observed and predicted SNV rates from the random forest model $(r=0.28 ; p$ value $<10^{-15}$ ) suggests a significant influence of the features included but, overall, a moderate level of predictive accuracy. Modelling was less successful in predicting BRCA-EU SV breakpoint rates $(r=0.09$ between observed and predicted values) but also indicated a significant association with chromatin and sequence features ( $p$ value $<10^{-15}$ ) (Fig. 7b). However, even though the magnitude of effects is rather modest, the direction of associations is strikingly inverted for SNV and SV rates, such that the variables most strongly correlated with elevated SV rates (DNaseHS, replication timing, G-quadruplex content, GC a

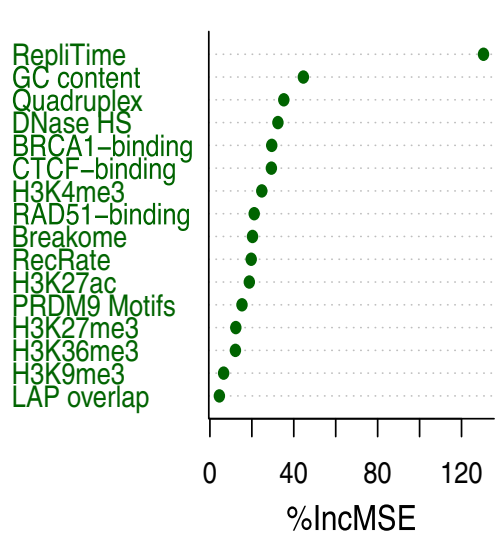

C

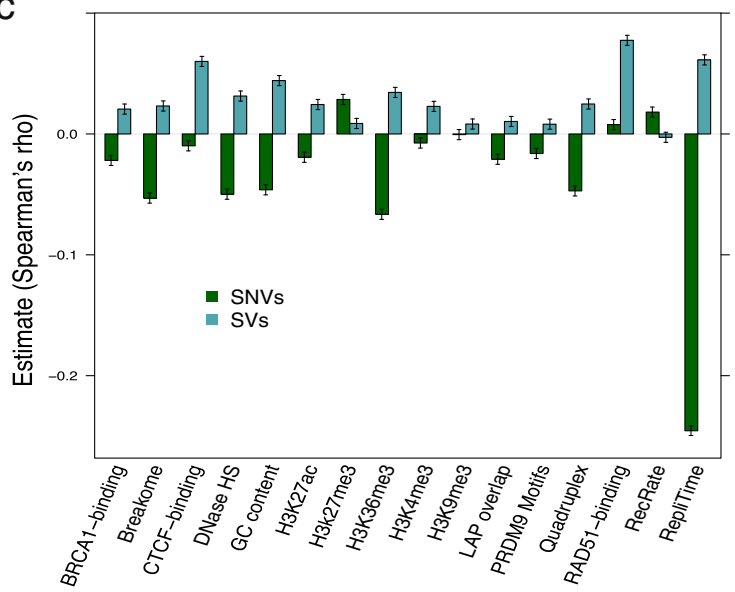

b

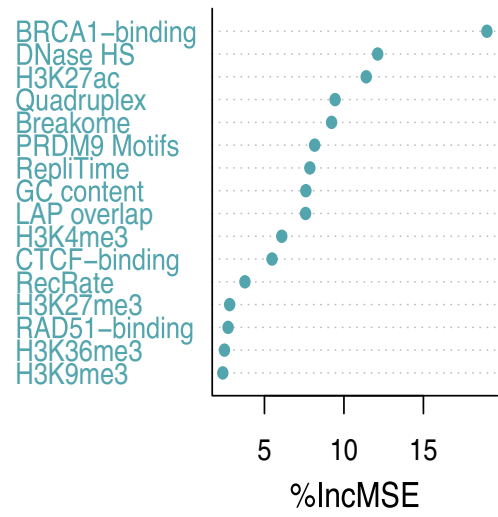

d

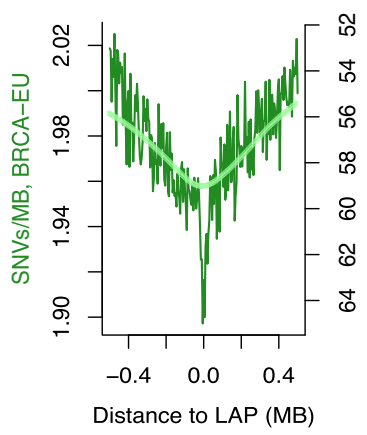

Fig. 7 Genomic and epigenomic features influencing SNV and SV rates at loop anchor points in breast tumours. a, b Variable importance, measured as percentage increase in mean squared error (\%IncMSE) when each predictor variable is removed from the model, predicting SNV rates (a) or SV breakpoint rates (b), respectively. c Spearman's rank correlation coefficient estimates between mutation rates and predictor variables of the random forest models, for SNV rates (green) and SV breakpoint rates (blue); $95 \%$ confidence intervals of the estimates are indicated. d SNV rates in BRCA-EU breast tumours within $500 \mathrm{~kb}$ of LAPs in the MCF-7 breast tumour cell line relative to replication timing (average MCF-7 ENCODE Repli-seq signal); higher Repli-seq values indicate earlier replication time 
content) are associated with decreased SNV rates (Fig. 7c). We conclude that similar chromatin and sequence features have moderate, but largely opposing, effects upon SNV and SV rates at LAPs.

Cell type-specific features are not available for most of the cancer types studied, but we carried out random forest regression analysis on mutation rates in each of the nine tumour types separately, using, as input features, only cell type-invariant features around the union set of 14,737 LAPs (Additional file 1: Table S3). As expected, the previously reported associations between mutation rates and replication timing, GC content and quadruplex sequence are seen across all tumour types. The estimates of the influence of overlapping LAPs (the variable "LAPs" in Additional file 1: Table S3) on mutation rates directly reflects the dip-or lack thereof-of mutation rates at LAPs seen in a given tumour type (Additional file 1: Figure S8). For example, in the case of melanoma, where a strong decline in SNV rates is evident, this variable influences mutation rates strongly as reflected in a high \%MSE score. In contrast, in malignant lymphoma, where the SNV decline at LAPs is very modest, LAP overlap has little measurable effect on SNV rates.

\section{Genes within recurrently disrupted chromatin loops are enriched for functions in the cell cycle}

Recent literature has documented tumours showing oncogene upregulation as a result of disrupted CTCF binding sites and chromatin loops in a variety of cancers [1]. Using breakpoint data from all 1672 ICGC donors and the union set of 14,737 CTCF-containing LAPs, we find that genes within the top $5 \%$ most disrupted chromatin loops (possessing five or more SV breakpoints in their LAPs) are enriched for functional annotation terms associated with proliferation and the G1/S cell cycle transition (Additional file 1: Table S4) [55]. The enrichments of such putatively cancer-associated terms are often only marginally significant given current sample sizes, but are broadly consistent with previously reported oncogenic disruptions [1]. Thus, it is possible that frequent disruptions of chromatin loops and domain boundaries in proximity to oncogenes in tumours are driven by the unusual mutational biases at LAPs.

In the breast cancer dataset, we also observe an unexpected excess of overlap between recurrently disrupted HMEC loops (bordered by LAPs that are disrupted in at least two samples) and GWAS regions associated with breast cancer: there were 40 such overlaps, whereas only 18.1 overlaps were, on average, observed in 5000 circular permutations $(p=0.0002)$. This excess in overlap is notably larger than that observed for the background set of all HMEC loops and GWAS hits (225 observed overlaps and a mean of 143.6 expected overlaps, based on 5000 permutations; $p=0.001$ ), suggesting a possible causal relationship between LAP disruption and the breast cancer phenotype.

\section{Common fragile sites and LAPs}

Common fragile sites (CFSs) are large, initially cytogenetically defined, genomic regions characterized by high rates of DNA breakage, active transcription and late replication [56, 57]. Interestingly, we found more LAP-gene intersections for protein-coding genes within CFSs compared to LAP-gene intersections in the genome as a whole $(0.77$ and 0.47 LAPs per gene; relative proportion test in $\mathrm{R} p<10^{-6}$ ), while the average replication timing was indistinguishable between the two sets of LAPs (Wilcoxon test in $R$, not significant). If genes are more often interrupted by LAPs in fragile regions, this leaves the intriguing possibility that at least a subset of CFSs are caused by DNA looping and the instability associated with LAPs.

\section{Discussion}

LAPs and recombination HSs are two seemingly unrelated features of the genome-one involved in chromatin organisation and the other in recombination during meiosis-but both classes of sites emerge as hotspots for DSBs. We have shown that LAPs and HSs often occur in the same genomic locations, which suggests that the same genomic regions that migrate to the chromosomal axis during meiosis, ultimately forming the points of breakage for DSB initiation [58], are also involved in chromatin organisation in the interphase nucleus of somatic cells. Intriguingly, cohesin, which associates with CTCF at LAPs $[59,60]$, is also enriched at the meiotic loop axis and plays a diverse role in chromosome pairing in both mitosis and meiosis [61]. Coincidentally, a recent study has shown that a subset of PRDM9 binding occurs at CTCF sites in mouse spermatocytes, and an interaction between the two proteins has been suggested [62]. This observation is consistent with an enrichment of recombination HSs at CTCF- LAPs (but not at non-CTCF LAPs), while the enrichment of PRDM9 motifs within CTCF-LAPs also suggests a sequence-based mechanism. A variety of factors, many related to chromatin structure, affect the propensity of LAPs to harbour SV breakpoints. PRDM9 also appears to be active in at least a subset of cancer cells [50] and may contribute to DSB formation at LAPs, suggesting another possible link between LAPs and HSs. Further, the association of LAPs with DSB formation appears to be at least partly attributable to the enrichment of active promoters and enhancers at LAPs, which is consistent with reports that promoters are inherently prone to DSBs, in both somatic cells [41] and meiotic cells that lack PRDM9 [63]. However, we also observe an excess of DSBs at LAPs that have no overlap with promoter or enhancer states, demonstrating that DNA breakage is not solely due to these 
elements. LAPs are unusual with respect to replication timing, with LAPs replicating, on average, earlier than their surrounding regions, consistent with the dual roles of cohesin in stabilising chromatin loops and also initiating replication [64]. Accordingly, chromatin looping may, to some extent, directly result from the initiation of replication or, conversely, determine its starting position in the next cell cycle [65]. Given the strong association between LAPs and DSB-prone regions, disruption of replication near such origins may be one way in which genome instability is introduced in cancer [66]. Notably, regions stably bound by DNA binding proteins such as CTCF seem to suffer high mutational loads due to replication errors [27].

LAPs are foci of DSB breakpoints and may provide the raw material for cancer evolution via structural variation, dependent on other factors, such as deficiencies in DNA repair pathways. The resulting SVs may have been subject to selection in cancer, though the majority are likely to be 'passenger' variants that drift toward fixation with little phenotypic consequences in their tissue of origin. Accordingly, we observe a strong enrichment of somatic DSB formation at LAPs in the NHEK cell line, more modest elevations in SV breakpoints around LAPs in cancers, and only some evidence that the genes affected are those that experience selection in cancers. At the human population level, our results suggest that chromatin loops are predominantly inherited as a genetic unit, with recombination often confined to LAPs, and therefore tending to preserve regulatory haplotypes. Consistent with our results, recent studies have shown that linkage disequilibrium (LD) blocks are enriched within TADs, i.e. recombination between enhancers and their target genes is reduced within domains [67]. Indeed, HSs themselves may primarily be a by-product of particular chromatin environments, replication timing and other functional constraints, such as a lack of active transcription, which may interfere with the recombination process [68].

We have used aggregate analysis across loop anchor points and cancer types to show that a mutational bias towards somatic breakage of chromatin loop anchors exists, consistent with recent experimental data from mouse B cells [16]; notably, breakage is more prominent in some cancer types than others and presumably depends on the general genome instability of the tumour type. The unusual DNA breakage patterns near LAPs are likely to contribute to cancer evolution, reflected in higher SV breakpoint levels, and allowing for novel promoter-enhancer interactions. The increase in breakage near LAPs is influenced by their specific chromatin environment and replication timing, DNA folding and accessibility to the repair machinery. Similar influences may underlie the surprising association of LAPs with meiotic recombination events.

\section{Conclusions}

In this study, we show that chromatin loop anchor points are fragile sites in the genome, acquiring DNA breakage in a range of cellular contexts, such as in normal cell lines, in cancer and during meiosis. The implications of this are far-reaching, affecting gene regulation in somatic tissues as well as the modular structure of the genome during evolution.

\section{Methods}

\section{LAP and recombination HS datasets}

Chromatin loops for cell lines representing all human germ layers (GEO dataset GM12878, HeLa, HMEC, HUVEC, IMR90, K562 and NHEK) were derived from unusually high resolution in situ $\mathrm{Hi}-\mathrm{C}$ data, defining LAPs at a resolution of $1-5 \mathrm{~kb}$ (accession GSE63525) [19]. These loops are often conserved between cell lines, such that $55-75 \%$ of the loops detected in any given cell line were also found in the most deeply sequenced cell line (GM12878), and around 50\% appear to be conserved across mammalian species [19]. The majority of loops are also associated with convergently orientated CTCF binding motifs at the putative LAPs, consistent with the known roles of CTCF in loop formation [19]. On average, $17 \%$ of LAPs were only observed in one tissue (Additional file 1: Figure S5), with more deeply sequenced cell lines consistently resulting in more LAPs being called. From this dataset, we created a merged dataset of 14,737 LAPs, centred around their associated (and convergently orientated) CTCF motifs [19], which represents the union of LAPs across all cell lines. For this purpose, the seven files annotated as "looplist_with_motif" for human cell lines were downloaded from the GEO dataset GSE63525; the genomic locations of CTCF motifs assigned to LAPs were merged if overlapping, such that each motif was only counted once, and the flanking sequence extended to $5 \mathrm{~kb}$. This merged dataset was used for all analyses except for the breast cancer-specific analysis, where we used only LAPs derived from the HMEC (human mammary epithelial cells) $\mathrm{Hi}-\mathrm{C}$ data, and the DSB analysis, where tissue-matched NHEK (normal human epidermal keratinocyte) LAPs were used, both of which were provided by [19].

To establish a control set of CTCF sites that were presumably not acting as loop anchors, we intersected CTCF motifs found in constitutively open chromatin [21] with all LAPs of the Rao et al. [19] dataset (plus $10 \mathrm{~kb}$ of flanking region), which resulted in 1845 constitutively bound CTCF motifs that were not found near LAPs, 1300 of which were in a uniquely mappable 
sequence context. We want to highlight, however, that this dataset may contain many false negatives, i.e. CTCF sites that act as anchor points in undetected LAPs.

Conversely, we used a union set of 11,890 merged LAPs for which a localized CTCF motif could not be found as control non-CTCF LAPs. However, since Rao et al. [19] had used very stringent search criteria for CTCF anchor sites, such as requiring SMC3/RAD21 binding, we conservatively removed from this dataset 9539 LAPs in which we detected a CTCF motif, using default search parameters in FIMO [69]. A further 249 of the remaining LAPs were bound by CTCF in encode datasets [70], leaving a total of 2102 conservatively called non-CTCF LAPs, 1578 of which were in a uniquely mappable sequence context. The sets of non-LAP CTCF sites and non-CTCF LAPs were used to create Additional file 1: Figure S4. To test for an enrichment of other transcription factor binding motifs at non-CTCF LAPs, we intersected the genomic coordinates of these LAPs with the locations of 118 motifs found in constitutively open chromatin [21].

Recombination hotspot locations had been identified in the phase II HapMap dataset (release 21) [71, 72]; recombination rates and SNP data were derived from phase 3 of the 1000 Genomes Project [47]. For the mutation rate analysis, we created $1-\mathrm{Mb}$ windows around the midpoints of LAPs and HSs and omitted regions containing ENCODE blacklisted genomic regions (http://hgdownload.cse.ucsc.edu/goldenPath/hg19/encodeDCC/wgEncodeMapability), resulting in 11,085 union LAPs, 6214 HMEC LAPs, and 18,914 recombination HSs plus their respective flanking regions. Median distances between the centre points of adjacent features were 106,331 bp for union LAPs, 125,000 bp for HMEC LAPs and $55,500 \mathrm{bp}$ for HSs, respectively. We divided each $1-\mathrm{Mb}$ region around a LAP into 5-kb non-overlapping windows; measures of mutation rates and overlap with other genomic features were calculated for each 5-kb bin.

\section{Cancer mutation rates}

Release_23 ICGC (https://dcc.icgc.org/) datasets with whole-genome sequence-derived calls for both SNV and SVs (not under publication moratorium in April 2017) were included: BOCA-FR (bone cancer), BRCA-EU (breast cancer), CLLE-ES (chronic lymphocytic leukemia), LIRI-JP (liver cancer), MALY-DE (malignant lymphoma), MELA-AU (skin cancer), OV-AU (ovarian cancer), PACA-AU (pancreatic cancer), PAEN-AU (pancreatic cancer), PAEN-IT (pancreatic cancer), EOPC-DE (early onset prostate cancer), PRAD-CA (prostate cancer), PRAD-UK (prostate cancer). The combined analysis included a total of 32,105,808 SNVs and 368,480 structural variants (Additional file 1: Table S1). We included all categories of structural variants that were listed in the ICGC files (i.e. insertions, deletions, inversions etc.), recording all breakpoint positions based on the coordinates of the SVs (i.e. a single SV has two breakpoint positions). SNV and breakpoint rates were intersected with the genomic coordinates of LAPs and HSs. Confidence intervals (as shown in Fig. 1) were calculated based on the assumption that breakpoint and SNV rates are random processes and follow the Poisson distribution: for this purpose, the aggregate number of mutations over all samples and $5-\mathrm{kb}$ windows was calculated. This number is of the order of half a million for SNVs and $\sim 8000$ for SVs. For example, a 5 -kb window with $\sim 4$ SNVs per ICGC sample per megabase contains a total aggregate number of 506,363 SNVs $(4 \mathrm{SNVs} \times 5 / 1000 \times 2284$ samples $\times 11,085$, the number of times a window was sampled). The corresponding $95 \%$ confidence interval for the aggregate number of SNVs was calculated using the poisson.test() function in R, in the example as [504,970, 507,760].

The $R$ package SomaticSignatures [73] was used to calculate mutational signatures at union CTCF-LAPs, non-CTCF LAPS and CTCF motifs for each tissue type separately.

\section{Genomic features near LAPs and recombination HSs}

Germline de novo mutation rates were reported for a whole genome sequencing study of 283 Icelandic trios [38]. A range of genomic features were generated by the ENCODE consortium [74], including average replication timing for each 5-kb genomic window, which was calculated based on the Repli-seq wavelet-smoothed signal for MCF-7 (breast cancer) and HepG2 (liver cancer) cell lines; open chromatin sites (DNAse hypersensitivity), CTCF-binding in MCF-7; RAD51-binding in MCF-7; Broad chromHMM tracks for chromatin state segmentation of HMEC; histone modifications (H3K27ac, H3K09me3, H3K4me3, H3K36me3 and H3K27me3) in MCF-7 and HepG2; nucleosome occupancy scores for GM12878. Genomic features based on ChIP-seq data were represented as genomic segments (peaks called) in the ENCODE distributed files; the overlap (in base pairs) between these features and genomic windows around LAPs was calculated using bedtools [75]. GC content for each 5-kb window was also calculated using bedtools [75]. Sites predicted to adopt quadruplex conformations were generated by Kudlicki [76] (http://moment.utmb.edu/allquads/). DSBs were detected using the DSBCapture protocol in the NHEK cell line (GEO database accession GSE78172) [41], and, as in Lensing et al. [41], we used the intersection of both biological replicates as a high confidence DSB peak set. ChIP-seq data for BRCA1 binding in MCF-10A cells were generated by Gardini et al. [77] and MACS2 [78] was used to call peaks in BRCA1 binding using default parameters.

Average replication time was calculated from the Wavelet-smoothed Signals of the 15 Encode cell types 
available at the UCSC Genome Browser site (http://genome.ucsc.edu/).

\section{Random forest regression analysis of mutation rates}

Random forest regression models were constructed using the $R$ package randomForest [79]. To construct a model with 200 trees, we extracted genomic regions within $1 \mathrm{Mb}$ of a HMEC LAP, merging overlapping regions, i.e. counting each unique genomic region once. Response variables were the number of BRCA-EU SNVs or SVs per 5-kb window, for a total of 239,141 windows. Predictor variables were replication timing in MCF-7; GC content; quadruplex overlap; HapMap recombination rate; DSB regions in NHEK cells; MCF-7 DNAse hypersensitivity; BRCA1-binding in MCF10A; RAD51-binding in MCF-7; CTCF-binding in MCF-7; PRDM9 motif coverage; overlap with peaks of H3K4me3, H3K27ac, H3K36me3, H3K27me3 and H3K9me3 for MCF7 and MCF-10A cell lines; HMEC LAP presence.

\section{Random forest regression analysis of BRCA $1 / 2$ binding}

To model the outcome variables 'BRCA1 binding' and 'RAD51 binding' in MCF-10A and MCF-7 cell lines, respectively, we divided the genome up into $5-\mathrm{kb}$ windows around $1 \mathrm{Mb}$ of HMEC LAPs. Input features to the model were all 15 Broad chromHMM states for state segmentation in HMEC cells as well as the overlap with HMEC LAPs. Two-hundred random forest trees were constructed.

\section{LAP and recombination HS overlap}

Circular permutation within the $R$ package RegioneR [80] was used to assess the significance of genome-wide overlap between LAPs and recombination HS, using 100,000 permutations. The FIMO algorithm [69] from the MEME package [81] was used to scan the genome for occurrences of the 13-bp PRDM9 motif CCTC CCTNNCCAC, using default parameters; this resulted in 51,107 motif locations being identified with a motif match $p$ value $<1.3 \mathrm{e}-06$.

\section{Functional enrichment analysis of recurrently disrupted LAPs}

Functional annotation enrichment analysis was carried out for regions of interest using the GREAT tool to calculate FDR-corrected hypergeometric q-values for the default selection of annotation ontologies [82]. As the background set for enrichment analyses, we used the 9973 genomic regions encompassed by all loops within the union set of LAPs. As the foreground set, we used 398 genomic regions encompassed by LAPs disrupted five or more times across tumour samples, corresponding to the top $5 \%$ most disrupted loops.

Breast cancer associated SNPs were obtained from the GWAS catalogue [83] (2017-05-29 release) and their coordinates were extended by $5 \mathrm{~kb}$ (to account for LD tagging of nearby causal SNPs) according to the average span of LD blocks in 1000 Genomes Project data for European populations [84]. The resulting GWAS SNP-containing segments were merged using bedtools [75] to create a non-redundant set of GWAS regions; circular permutations were carried out in $R$ to test for an excess of overlap between the GWAS regions and chromatin loops in the HMEC cell line.

\section{Common fragile sites dataset}

The genomic locations of 70 protein-coding genes within CFSs (annotated as "common fragile sites") were downloaded from the NCBI Gene database.

\section{Programming languages}

Datasets were downloaded and formatted using unix shell scripting. Manuscript figures were created using custom scripts in $R$ [85].

\section{Additional files}

Additional file 1: Chromatin loop anchors are associated with genome instability in cancer and recombination hotspots in the germline. Figures S1-S8 and Tables S1-S4. (PDF 641 kb)

Additional file 2: Review history of this manuscript. (DOCX $199 \mathrm{~kb}$ )

\section{Acknowledgements}

We are indebted to the ICGC for the timely public release of tumour whole genome sequencing data. We wish to thank those who reviewed the manuscript for their constructive comments (Additional file 2).

\section{Funding}

This study was funded by core funding of the UK Medical Research Council (MRC) to the MRC Human Genetics Unit.

\section{Availability of data and materials}

All data used in this study are publicly available, from sources indicated in the manuscript. These include the GEO dataset GM12878, GSE78172 and GSE40591, which provide Hi-C data [19], DSBCapture experiments [41] and BRCA1-binding in MCF10A cells [77], respectively. The ensembl regulatory build [70] at http://www.ensembl.org/info/genome/funcgen/regulatory_build.html was used to find CTCF-bound genomic sites. Recombination hotspot locations came from HapMap phase II (release 21) at ftp://

ftp.ncbi.nlm.nih.gov/hapmap/recombination/2006-10_rel21_phasel+II/hotspots/ $[71,72]$ and population-level SNP rates from phase 3 of the $1000 \mathrm{Ge}$ nomes Project at http://www.internationalgenome.org/category/phase-3/ [47]. Somatic mutations were downloaded from the ICGC Release_23, available at https://dcc.icgc.org/. Germline de novo mutations are available at https://doi.org/10.1371/journal.pgen.1006315.s004 [38]. ENCODE datasets are available at http://hgdownload.cse.ucsc.edu/goldenpath/hg19/encodeDCC/ [74], predicted quadruplex sequences at http://moment.utmb.edu/allquads/ [76] and the GWAS catalogue (2017-05-29 release) at https://www.ebi.ac.uk/ gwas/docs/file-downloads [83].

\section{Authors' contributions}

The study was conceived and the manuscript written by VBK and CAS. The data were analyzed by VBK. Both authors read and approved the final manuscript.

Ethics approval and consent to participate Not applicable. 


\section{Consent for publication}

Not applicable.

\section{Competing interests}

The authors declare that they have no competing interests.

\section{Publisher's Note}

Springer Nature remains neutral with regard to jurisdictional claims in published maps and institutional affiliations.

\section{Received: 14 December 2017 Accepted: 13 July 2018}

\section{Published online: 30 July 2018}

\section{References}

1. Kaiser VB, Semple CA. When TADs go bad: chromatin structure and nuclear organisation in human disease. F1000Res. 2017;6:F1000.

2. Lupianez DG, Spielmann M, Mundlos S. Breaking TADs: how alterations of chromatin domains result in disease. Trends Genet. 2016;32:225-37.

3. Peifer M, Hertwig F, Roels F, Dreidax D, Gartlgruber M, Menon R, Kramer A, Roncaioli JL, Sand F, Heuckmann JM, et al. Telomerase activation by genomic rearrangements in high-risk neuroblastoma. Nature. 2015;526:700-4.

4. Valentijn LJ, Koster J, Zwijnenburg DA, Hasselt NE, van Sluis P, Volckmann R, van Noesel MM, George RE, Tytgat GA, Molenaar JJ, Versteeg R. TERT rearrangements are frequent in neuroblastoma and identify aggressive tumors. Nat Genet. 2015;47:1411-4.

5. Northcott PA, Lee C, Zichner T, Stutz AM, Erkek S, Kawauchi D, Shih DJ, Hovestadt V, Zapatka M, Sturm D, et al. Enhancer hijacking activates GFI1 family oncogenes in medulloblastoma. Nature. 2014;511:428-34.

6. Groschel S, Sanders MA, Hoogenboezem R, de Wit E, Bouwman BA Erpelinck C, van der Velden VH, Havermans M, Avellino R, van Lom K, et al. A single oncogenic enhancer rearrangement causes concomitant EVI1 and GATA2 deregulation in leukemia. Cell. 2014;157:369-81.

7. Hnisz D, Weintraub AS, Day DS, Valton AL, Bak RO, Li CH, Goldmann J, Lajoie BR, Fan ZP, Sigova AA, et al. Activation of proto-oncogenes by disruption of chromosome neighborhoods. Science. 2016:351:1454-8.

8. Weischenfeldt J, Dubash T, Drainas AP, Mardin BR, Chen Y, Stutz AM, Waszak SM, Bosco G, Halvorsen AR, Raeder B, et al. Pan-cancer analysis of somatic copy-number alterations implicates IRS4 and IGF2 in enhancer hijacking. Nat Genet. 2017:49:65-74.

9. Valton AL, Dekker J. TAD disruption as oncogenic driver. Curr Opin Genet Dev. 2016;36:34-40.

10. Dixon JR, Selvaraj S, Yue F, Kim A, Li Y, Shen Y, Hu M, Liu JS, Ren B. Topological domains in mammalian genomes identified by analysis of chromatin interactions. Nature. 2012:485:376-80.

11. Moore BL, Aitken S, Semple CA. Integrative modeling reveals the principles of multi-scale chromatin boundary formation in human nuclear organization. Genome Biol. 2015;16:110.

12. Splinter E, Heath H, Kooren J, Palstra RJ, Klous P, Grosveld F, Galjart N, de Laat W. CTCF mediates long-range chromatin looping and local histone modification in the beta-globin locus. Genes Dev. 2006;20:2349-54.

13. Haarhuis JHI, van der Weide RH, Blomen VA, Yanez-Cuna JO, Amendola M, van Ruiten MS, Krijger PHL, Teunissen $H$, Medema $\mathrm{RH}$, van Steensel $B$, et al. The cohesin release factor WAPL restricts chromatin loop extension. Cell. 2017;169:693-707. e614

14. Fudenberg G, Imakaev M, Lu C, Goloborodko A, Abdennur N, Mirny LA. Formation of chromosomal domains by loop extrusion. Cell Rep. 2016;15:2038-49.

15. Nichols $\mathrm{MH}$, Corces VG. A CTCF code for 3D genome architecture. Cell. 2015;162:703-5.

16. Canela A, Maman Y, Jung S, Wong N, Callen E, Day A, Kieffer-Kwon KR, Pekowska A, Zhang H, Rao SSP, et al. Genome organization drives chromosome fragility. Cell. 2017;170:507-21. e518

17. Uuskula-Reimand L, Hou H, Samavarchi-Tehrani P, Rudan MV, Liang M, Medina-Rivera A, Mohammed H, Schmidt D, Schwalie P, Young EJ, et al. Topoisomerase II beta interacts with cohesin and CTCF at topological domain borders. Genome Biol. 2016;17:182.

18. Manville CM, Smith K, Sondka Z, Rance H, Cockell S, Cowell IG, Lee KC, Morris NJ, Padget K, Jackson GH, Austin CA. Genome-wide ChIP-seq analysis of human TOP2B occupancy in MCF7 breast cancer epithelial cells. Biol Open. 2015;4:1436-47.

19. Rao SS, Huntley MH, Durand NC, Stamenova EK, Bochkov ID, Robinson JT, Sanborn AL, Machol I, Omer AD, Lander ES, Aiden EL. A 3D map of the human genome at kilobase resolution reveals principles of chromatin looping. Cell. 2014:159:1665-80.

20. Nora EP, Goloborodko A, Valton AL, Gibcus JH, Uebersohn A, Abdennur N Dekker J, Mirny LA, Bruneau BG. Targeted degradation of CTCF decouples local insulation of chromosome domains from genomic compartmentalization. Cell. 2017:169:930-44. e922

21. Kaiser VB, Taylor MS, Semple CA. Mutational biases drive elevated rates of substitution at regulatory sites across cancer types. PLoS Genet. 2016;12: e1006207.

22. Katainen R, Dave K, Pitkanen E, Palin K, Kivioja T, Valimaki N, Gylfe AE, Ristolainen $\mathrm{H}$, Hanninen UA, Cajuso T, et al. CTCF/cohesin-binding sites are frequently mutated in cancer. Nat Genet. 2015;47:818-21.

23. Flavahan WA, Drier Y, Liau BB, Gillespie SM, Venteicher AS, StemmerRachamimov AO, Suva ML, Bernstein BE. Insulator dysfunction and oncogene activation in IDH mutant gliomas. Nature. 2016:529:110-4.

24. Stamatoyannopoulos JA, Adzhubei I, Thurman RE, Kryukov GV, Mirkin SM, Sunyaev SR. Human mutation rate associated with DNA replication timing. Nat Genet. 2009;41:393-5.

25. Dileep V, Ay F, Sima J, Vera DL, Noble WS, Gilbert DM. Topologically associating domains and their long-range contacts are established during early G1 coincident with the establishment of the replication-timing program. Genome Res. 2015;25:1104-13.

26. Schuster-Bockler B, Lehner B. Chromatin organization is a major influence on regional mutation rates in human cancer cells. Nature. 2012;488:504-7.

27. Reijns MA, Kemp H, Ding J, de Proce SM, Jackson AP, Taylor MS. Laggingstrand replication shapes the mutational landscape of the genome. Nature. 2015;518:502-6.

28. Sabarinathan R, Mularoni L, Deu-Pons J, Gonzalez-Perez A, Lopez-Bigas N. Nucleotide excision repair is impaired by binding of transcription factors to DNA. Nature. 2016:532:264-7.

29. Perera D, Poulos RC, Shah A, Beck D, Pimanda JE, Wong JW. Differential DNA repair underlies mutation hotspots at active promoters in cancer genomes. Nature. 2016;532:259-63.

30. Drier Y, Lawrence MS, Carter SL, Stewart C, Gabriel SB, Lander ES, Meyerson M, Beroukhim R, Getz G. Somatic rearrangements across cancer reveal classes of samples with distinct patterns of DNA breakage and rearrangement-induced hypermutability. Genome Res. 2013;23:228-35.

31. Engreitz JM, Agarwala V, Mirny LA. Three-dimensional genome architecture influences partner selection for chromosomal translocations in human disease. PLoS One. 2012;7:e44196.

32. Fudenberg G, Getz G, Meyerson M, Mirny LA. High order chromatin architecture shapes the landscape of chromosomal alterations in cancer. Nat Biotechnol. 2011:29:1109-13.

33. Carvalho CM, Lupski JR. Mechanisms underlying structural variant formation in genomic disorders. Nat Rev Genet. 2016:17:224-38.

34. Coop G, Wen X, Ober C, Pritchard JK, Przeworski M. High-resolution mapping of crossovers reveals extensive variation in fine-scale recombination patterns among humans. Science. 2008;319:1395-8.

35. Myers S, Bottolo L, Freeman C, McVean G, Donnelly P. A fine-scale map of recombination rates and hotspots across the human genome. Science. 2005:310:321-4.

36. Hicks WM, Kim M, Haber JE. Increased mutagenesis and unique mutation signature associated with mitotic gene conversion. Science. 2010:329:82-5.

37. Strathern JN, Shafer BK, McGill CB. DNA synthesis errors associated with double-strand-break repair. Genetics. 1995:140:965-72.

38. Besenbacher $S$, Sulem P, Helgason A, Helgason H, Kristjansson H, Jonasdottir A, Jonasdottir A, Magnusson OT, Thorsteinsdottir U, Masson G, et al. Multinucleotide de novo Mutations in Humans. PLoS Genet. 2016;12:e1006315.

39. International Cancer Genome C, Hudson TJ, Anderson W, Artez A, Barker AD, Bell C, Bernabe RR, Bhan MK, Calvo F, Eerola l, et al. International network of cancer genome projects. Nature. 2010;464:993-8.

40. Morganella S, Alexandrov LB, Glodzik D, Zou X, Davies H, Staaf J, Sieuwerts AM, Brinkman AB, Martin S, Ramakrishna M, et al. The topography of mutational processes in breast cancer genomes. Nat Commun. 2016;7:11383.

41. Lensing SV, Marsico G, Hansel-Hertsch R, Lam EY, Tannahill D, Balasubramanian S. DSBCapture: in situ capture and sequencing of DNA breaks. Nat Methods. 2016:13:855-7.

42. Hänsel-Hertsch R, Di Antonio M, Balasubramanian S. DNA G-quadruplexes in the human genome: detection, functions and therapeutic potential. Nat Rev Mol Cell Biol. 2017;18:279-84. 
43. Deng $\mathrm{CX}$, Wang $\mathrm{RH}$ : Roles of BRCA1 in DNA damage repair: a link between development and cancer. Hum Mol Genet 2003; 12 Spec No 1:R113-R123.

44. Lord CJ, Ashworth A. The DNA damage response and cancer therapy. Nature. 2012:481:287-94.

45. Walsh CS. Two decades beyond BRCA1/2: Homologous recombination, hereditary cancer risk and a target for ovarian cancer therapy. Gynecol Oncol. 2015;137:343-50.

46. Hatchi E, Skourti-Stathaki K, Ventz S, Pinello L, Yen A, Kamieniarz-Gdula K, Dimitrov S, Pathania S, McKinney KM, Eaton ML, et al. BRCA1 recruitment to transcriptional pause sites is required for R-loop-driven DNA damage repair. Mol Cell. 2015;57:636-47.

47. The 1000 Genomes Project Consortium, Auton A, Brooks LD, Durbin RM, Garrison EP, Kang HM, Korbel JO, Marchini JL, McCarthy S, McVean GA Abecasis GR. A global reference for human genetic variation. Nature. 2015; 526:68-74

48. Myers S, Bowden R, Tumian A, Bontrop RE, Freeman C, MacFie TS, McVean $G$, Donnelly P. Drive against hotspot motifs in primates implicates the PRDM9 gene in meiotic recombination. Science. 2010;327:876-9.

49. Pratto F, Brick K, Khil P, Smagulova F, Petukhova GV, Camerini-Otero RD. DNA recombination. Recombination initiation maps of individual human genomes. Science. 2014;346:1256442.

50. Feichtinger J, Aldeailej I, Anderson R, Almutairi M, Almatrafi A, Alsiwiehri N, Griffiths K, Stuart N, Wakeman JA, Larcombe L, McFarlane RJ. Meta-analysis of clinical data using human meiotic genes identifies a novel cohort of highly restricted cancer-specific marker genes. Oncotarget. 2012;3:843-53.

51. Charlesworth B. Fundamental concepts in genetics: effective population size and patterns of molecular evolution and variation. Nat Rev Genet. 2009:10:195-205.

52. Hilmi K, Jangal M, Marques M, Zhao T, Saad A, Zhang C, Luo VM, Syme A, Rejon C, Yu Z, et al. CTCF facilitates DNA double-strand break repair by enhancing homologous recombination repair. Sci Adv. 2017;3:e1601898.

53. Nik-Zainal S, Morganella S. Mutational signatures in breast cancer: the problem at the DNA level. Clin Cancer Res. 2017;23:2617-29.

54. Taylor BJ, Nik-Zainal S, Wu YL, Stebbings LA, Raine K, Campbell PJ, Rada C, Stratton MR, Neuberger MS. DNA deaminases induce break-associated mutation showers with implication of $\mathrm{APOBEC} 3 \mathrm{~B}$ and $3 \mathrm{~A}$ in breast cancer kataegis. Elife. 2013;2:e00534.

55. Bertoli C, Skotheim JM, de Bruin RA. Control of cell cycle transcription during G1 and S phases. Nat Rev Mol Cell Biol. 2013;14:518-28.

56. Glover TW, Wilson TE, Arlt MF. Fragile sites in cancer: more than meets the eye. Nat Rev Cancer. 2017;17:489-501.

57. Glover TW, Berger C, Coyle J, Echo B. DNA polymerase alpha inhibition by aphidicolin induces gaps and breaks at common fragile sites in human chromosomes. Hum Genet. 1984;67:136-42.

58. Baudat $F$, Imai $Y$, de Massy B. Meiotic recombination in mammals: localization and regulation. Nat Rev Genet. 2013;14:794-806.

59. Tang Z, Luo OJ, Li X, Zheng M, Zhu JJ, Szalaj P, Trzaskoma P, Magalska A Wlodarczyk J, Ruszczycki B, et al. CTCF-mediated human 3D genome architecture reveals chromatin topology for transcription. Cell. 2015;163:1611-27.

60. Ong CT, Corces VG. CTCF: an architectural protein bridging genome topology and function. Nat Rev Genet. 2014;15:234-46.

61. McNicoll F, Stevense M, Jessberger R. Cohesin in gametogenesis. Curr Top Dev Biol. 2013;102:1-34.

62. Grey C, Clement JA, Buard J, Leblanc B, Gut I, Gut M, Duret L, de Massy B. In vivo binding of PRDM9 reveals interactions with noncanonical genomic sites. Genome Res. 2017;27:580-90.

63. Brick K, Smagulova F, Khil P, Camerini-Otero RD, Petukhova GV. Genetic recombination is directed away from functional genomic elements in mice. Nature. 2012:485:642-5.

64. Guillou E, Ibarra A, Coulon V, Casado-Vela J, Rico D, Casal I, Schwob E, Losada A, Mendez J. Cohesin organizes chromatin loops at DNA replication factories. Genes Dev. 2010;24:2812-22.

65. Courbet S, Gay S, Arnoult N, Wronka G, Anglana M, Brison O, Debatisse M. Replication fork movement sets chromatin loop size and origin choice in mammalian cells. Nature. 2008;455:557-60.

66. Losada A. Cohesin in cancer: chromosome segregation and beyond. Nat Rev Cancer. 2014;14:389-93.

67. Liu Y, Sarkar A, Kheradpour P, Ernst J, Kellis M. Evidence of reduced recombination rate in human regulatory domains. Genome Biol. 2017;18:193.

68. McVicker G, Green P. Genomic signatures of germline gene expression. Genome Res. 2010;20:1503-11.
69. Grant CE, Bailey TL, Noble WS. FIMO: scanning for occurrences of a given motif. Bioinformatics. 2011:27:1017-8.

70. Zerbino DR, Wilder SP, Johnson N, Juettemann T, Flicek PR. The ensembl regulatory build. Genome Biol. 2015;16:56

71. McVean GA, Myers SR, Hunt S, Deloukas P, Bentley DR, Donnelly P. The finescale structure of recombination rate variation in the human genome. Science. 2004;304:581-4.

72. Winckler W, Myers SR, Richter DJ, Onofrio RC, McDonald GJ, Bontrop RE, McVean GA, Gabriel SB, Reich D, Donnelly P, Altshuler D. Comparison of fine-scale recombination rates in humans and chimpanzees. Science. 2005; 308:107-11.

73. Gehring JS, Fischer B, Lawrence M, Huber W. SomaticSignatures: inferring mutational signatures from single-nucleotide variants. Bioinformatics. 2015; 31:3673-5.

74. Encode Project Consortium. An integrated encyclopedia of DNA elements in the human genome. Nature. 2012;489:57-74.

75. Quinlan AR, Hall IM. BEDTools: a flexible suite of utilities for comparing genomic features. Bioinformatics. 2010;26:841-2.

76. Kudlicki AS. G-quadruplexes involving both strands of genomic DNA are highly abundant and colocalize with functional sites in the human genome. PLoS One. 2016;11:e0146174

77. Gardini A, Baillat D, Cesaroni M, Shiekhattar R. Genome-wide analysis reveals a role for BRCA1 and PALB2 in transcriptional co-activation. EMBO J. 2014; 33:890-905.

78. Zhang Y, Liu T, Meyer CA, Eeckhoute J, Johnson DS, Bernstein BE, Nusbaum C, Myers RM, Brown M, Li W, Liu XS. Model-based analysis of ChIP-Seq (MACS). Genome Biol. 2008;9:R137.

79. Liaw A, Wiener M. Classification and regression by randomForest. R News. 2002;2:18-22.

80. Gel B, Diez-Villanueva A, Serra E, Buschbeck M, Peinado MA, Malinverni R. regioneR: an $\mathrm{R} /$ Bioconductor package for the association analysis of genomic regions based on permutation tests. Bioinformatics. 2016;32:289-91.

81. Bailey TL, Boden M, Buske FA, Frith M, Grant CE, Clementi L, Ren J, Li WW, Noble WS. MEME SUITE: tools for motif discovery and searching. Nucleic Acids Res. 2009;37:W202-8.

82. McLean CY, Bristor D, Hiller M, Clarke SL, Schaar BT, Lowe CB, Wenger AM, Bejerano G. GREAT improves functional interpretation of cis-regulatory regions. Nat Biotechnol. 2010;28:495-501.

83. MacArthur J, Bowler E, Cerezo M, Gil L, Hall P, Hastings E, Junkins H, McMahon A, Milano A, Morales J, et al. The new NHGRI-EBI Catalog of published genome-wide association studies (GWAS Catalog). Nucleic Acids Res. 2017·45:D896-901.

84. Rosenfeld JA, Mason CE, Smith TM. Limitations of the human reference genome for personalized genomics. PLoS One. 2012;7:e40294.

85. R Core Team. R: A language and environment for statistical computing. Vienna: R Foundation for Statistical Computing. 2013. http://www.R-project. org/

Ready to submit your research? Choose BMC and benefit from:

- fast, convenient online submission

- thorough peer review by experienced researchers in your field

- rapid publication on acceptance

- support for research data, including large and complex data types

- gold Open Access which fosters wider collaboration and increased citations

- maximum visibility for your research: over $100 \mathrm{M}$ website views per year

At $\mathrm{BMC}$, research is always in progress.

Learn more biomedcentral.com/submission 